\title{
On an additive function on the set of ideals of an arbitrary number field
}

\author{
by \\ TianXin CaI (Hangzhou)
}

1. Introduction. In [1] K. Alladi and P. Erdős showed that if $\beta_{\alpha}(n)=$ $\sum_{p \mid n} p^{\alpha}$ then

$$
\sum_{n \leq x} \beta_{1}(n) \sim \frac{\pi^{2}}{12} \cdot \frac{x^{2}}{\log x}
$$

Many papers have been written concerning the additive function $\beta_{1}(n)$, see for example [4], [5], [6]. The best result is due to the author [2] and A. Mercier [9]; they have given the asymptotic formula

$$
\sum_{n \leq x} \beta_{1}(n)=x^{2}\left\{\sum_{i=1}^{m} d_{i} / \log ^{i} x+O\left(1 / \log ^{m+1} x\right)\right\}
$$

with arbitrary fixed $m \geq 1$ and

$$
d_{i}=\left(\sum_{v=0}^{i-1} \frac{(-2)^{v}}{v !} \zeta^{(v)}(2)\right) \frac{(i-1) !}{2^{i}}, \quad 1 \leq i \leq m .
$$

J. M. De Koninck and A. Ivić [5] also got (1), but they did not give the expression of (2).

In 1989, P. Zarzycki [10] studied the distribution of values of an additive function $\mathcal{B}_{\alpha}(\mathfrak{a})$ on the Gaussian integers given by

$$
\mathcal{B}_{\alpha}(\mathfrak{a})=\sum_{\mathfrak{p} \mid \mathfrak{a}}^{*} N(\mathfrak{p})^{\alpha}
$$

with fixed $\alpha>0$; the asterisk means that the summation is over the nonassociate prime divisors $\mathfrak{p}$ of a Gaussian integer $\mathfrak{a}$ and $N(\mathfrak{a})=N(x+i y)=$ $x^{2}+y^{2}$ is the norm of $\mathfrak{a}$. This function is a generalization of the function $\beta_{\alpha}(n)$. Zarzycki obtained the asymptotic formula for the summatory function $\sum_{\mathfrak{a} \in D} \mathcal{B}_{\alpha}(\mathfrak{a})$ by using the complex integration technique where $D$ is a certain set of Gaussian integers. The main result he obtained is the following 
Theorem 1. For $x \rightarrow \infty$,

$$
\sum_{N(\mathfrak{a}) \leq x}^{*} \mathcal{B}_{\alpha}(\mathfrak{a})=\frac{\zeta(1+\alpha) L\left(1+\alpha, \chi_{4}\right)}{1+\alpha} \cdot \frac{x^{1+\alpha}}{\log x}\left(1+O\left(\frac{1}{\log x}\right)\right)
$$

where $\chi_{4}$ denotes the non-principal Dirichlet character modulo 4.

In [3], I announced that by using an elementary technique one could prove the following

Theorem 2. For $x \rightarrow \infty$,

$$
\begin{aligned}
\sum_{N(\mathfrak{a}) \leq x}^{*} \mathcal{B}_{\alpha}(\mathfrak{a})= & \sum_{n=1}^{N}\left(\sum_{v=0}^{n-1} \frac{(-1)^{v}(1+\alpha)^{v}}{v !} \xi^{(v)}(1+\alpha)\right) \\
& \times \frac{(n-1) ! x^{1+\alpha}}{(1+\alpha)^{n} \log ^{n} x}+O\left(\frac{x^{1+\alpha}}{\log ^{N+1} x}\right)
\end{aligned}
$$

with any fixed positive integer $N$, where $\xi(s)=\zeta(s) L\left(s, \chi_{4}\right)$ and $\xi^{(v)}(s)$ is the $v$-th derivative of $\xi(s)$.

In the present paper, we consider the function $\mathcal{B}_{\alpha}(\mathfrak{a})$ defined on the integral ideals of an arbitrary number field $K$ by the formula

$$
\mathcal{B}_{\alpha}(\mathfrak{a})=\sum_{\mathfrak{p} \mid \mathfrak{a}}^{*} N(\mathfrak{p})^{\alpha},
$$

where the asterisk means that $\mathfrak{p}$ runs over prime ideals of $K$. We obtain the following

Theorem 3. For every number field $K$,

$$
\begin{aligned}
\sum_{N(\mathfrak{a}) \leq x} \mathcal{B}_{\alpha}(\mathfrak{a})= & \sum_{n=1}^{N} \sum_{v=0}^{n-1} \frac{(-1)^{v}(1+\alpha)^{v}}{v !} \zeta_{K}^{(v)}(1+\alpha) \\
& \times \frac{(n-1) ! x^{1+\alpha}}{(1+\alpha)^{n} \log ^{n} x}+O\left(\frac{x^{1+\alpha}}{\log ^{N+1} x}\right)
\end{aligned}
$$

with any fixed positive integer $N$, where $\zeta_{K}$ is the Dedekind zeta function of $K$ and $\zeta_{K}^{(v)}$ its $v$-th derivative.

Clearly Theorem 3 contains Theorem 2 since every ideal of $\mathbb{Q}(\sqrt{-1})$ is principal.

\section{Auxiliary lemmas}

Lemma 1. Let $K$ be a number field. Then

$$
\pi(x, K):=\sum_{N(\mathfrak{p}) \leq x}^{*} 1=\operatorname{Li} x+O\left(x e^{-c \sqrt{\log x}}\right)
$$


with some $c>0$. Moreover, for arbitrary fixed $N \geq 1$,

$$
\pi(x, K)=\sum_{n=1}^{N} \frac{(n-1) ! x}{\log ^{n} x}+O\left(\frac{x}{\log ^{N+1} x}\right) .
$$

For the proof, see [8], Theorem 191, and [7], §4, formula (5).

Lemma 2. For arbitrary fixed $N \geq 1$ and $\alpha>0$,

$$
\sum_{N(\mathfrak{p}) \leq x}^{*} N(\mathfrak{p})^{\alpha}=\sum_{n=1}^{N} \frac{(n-1) !}{(1+\alpha)^{n}} \cdot \frac{x^{1+\alpha}}{\log ^{n} x}+O\left(\frac{x^{1+\alpha}}{\log ^{N+1} x}\right) .
$$

Proof. We have

$$
\begin{aligned}
\sum_{N(\mathfrak{p}) \leq x}^{*} N(\mathfrak{p})^{\alpha}= & \int_{2-\varepsilon}^{x} t^{\alpha} d \pi(t, K) \\
= & x^{\alpha} \pi(x, K)-\int_{2-\varepsilon}^{x} \alpha t^{\alpha-1} \pi(t, K) d t \\
= & \sum_{n=1}^{N} \frac{(n-1) ! x^{1+\alpha}}{\log ^{n} x}-\int_{2-\varepsilon}^{x} \sum_{n=1}^{N} \frac{(n-1) ! \alpha t^{\alpha}}{\log ^{n} t} d t+O\left(\frac{x^{1+\alpha}}{\log ^{N+1} x}\right) \\
= & \frac{1}{1+\alpha} \sum_{n=1}^{N} \frac{(n-1) ! x^{1+\alpha}}{\log ^{n} x}-\alpha \sum_{n=2}^{N}\left(\frac{1}{(1+\alpha)^{2}}+\frac{1}{(1+\alpha)^{3}}\right. \\
& \left.+\ldots+\frac{1}{(1+\alpha)^{n}}\right) \frac{(n-1) ! x^{1+\alpha}}{\log ^{n} x}+O\left(\frac{x^{1+\alpha}}{\log ^{N+1} x}\right) \\
= & \sum_{n=1}^{N} \frac{(n-1) !}{(1+\alpha)^{n}} \cdot \frac{x^{1+\alpha}}{\log ^{n} x}+O\left(\frac{x^{1+\alpha}}{\log ^{N+1} x}\right) \cdot \mathbf{-}
\end{aligned}
$$

Lemma 3. Let $2 \leq Q<\log ^{L+1} x$, for arbitrary fixed $L \geq 1$. Then

$$
\begin{aligned}
& \sum_{n=1}^{N} \frac{(}{(1+\alpha)^{n}} \cdot \frac{1}{\log ^{n} x / Q} \\
& \quad=\sum_{n=1}^{N}\left(\sum_{v=0}^{n-1} \frac{(1+\alpha)^{v} \log ^{v} Q}{v !}\right) \frac{(n-1) !}{(1+\alpha)^{n} \log ^{n} x}+O\left(\frac{\log ^{N} Q}{\log ^{N+1} x}\right) .
\end{aligned}
$$

Pr o of. By Newton's binomial formula,

$$
\sum_{n=1}^{N} \frac{(n-1) !}{(1+\alpha)^{n} \log ^{n} x / Q}=\sum_{n=1}^{N} \frac{(n-1) !}{(1+\alpha)^{n} \log ^{n} x}\left(1-\frac{\log Q}{\log x}\right)^{-n}
$$




$$
\begin{aligned}
& =\sum_{n=1}^{N} \frac{(n-1) !}{(1+\alpha)^{n} \log ^{n} x} \sum_{v=0}^{N-1}\left(\begin{array}{c}
-n \\
v
\end{array}\right)(-1)^{v}\left(\frac{\log Q}{\log x}\right)^{v}+O\left(\frac{\log ^{N} Q}{\log ^{N+1} x}\right) \\
& =\sum_{k=1}^{N} \frac{1}{\log ^{k} x} \sum_{\substack{n+v=k \\
n \geq 1, v \geq 0}}\left(\begin{array}{c}
n+v-1 \\
v
\end{array}\right) \frac{(n-1) !}{(1+\alpha)^{n}} \log ^{v} Q+O\left(\frac{\log ^{N} x}{\log ^{N+1} x}\right) \\
& =\sum_{k=1}^{N} \frac{(k-1) !}{(1+\alpha)^{k} \log ^{k} x} \sum_{v=0}^{k-1} \frac{(1+\alpha)^{v} \log ^{v} Q}{v !}+O\left(\frac{\log ^{N} Q}{\log ^{N+1} x}\right) .
\end{aligned}
$$

3. Proof of Theorem 3. Let $P(\mathfrak{a})$ denote a prime ideal factor of $\mathfrak{a}$ in $K$ of the largest possible norm. We should obtain the corresponding summation (3) for $N(P(\mathfrak{a}))^{\alpha}$ instead of $\mathcal{B}_{\alpha}(\mathfrak{a})$. In fact, let

$$
\sum_{N(\mathfrak{a}) \leq x}=\sum_{1}+\sum_{2}
$$

Here and below $\sum_{1}$ means $N(\mathfrak{a}) \leq x$ and $P(\mathfrak{a})$ is unique, $\sum_{2}$ means $N(\mathfrak{a})$ $\leq x$ and $P(\mathfrak{a})$ is not unique. By Theorem 202 of [8], one has

$$
\sum_{N(\mathfrak{a}) \leq x} 1=O(x)
$$

hence

(6) $\quad \sum_{2}\left(\mathcal{B}_{\alpha}(\mathfrak{a})-N(P(\mathfrak{a}))^{\alpha}\right)=O\left(x^{\alpha / 2} \log x\right) \sum_{N(\mathfrak{a}) \leq x} 1=O\left(x^{1+\alpha / 2} \log x\right)$.

On the other hand,

$$
\begin{aligned}
& \sum_{1}\left(\mathcal{B}_{\alpha}(\mathfrak{a})-N(P(\mathfrak{a}))^{\alpha}\right) \\
& =\sum_{1}\left\{\sum_{\substack{\mathfrak{p}^{k} \| \mathfrak{a}, \mathfrak{p}=P(\mathfrak{a}) \\
k \geq 2}}^{*} N(\mathfrak{p})^{\alpha}+\sum_{\mathfrak{p} \mid \mathfrak{a}, \mathfrak{p} \neq P(\mathfrak{a})}^{*} N(\mathfrak{p})^{\alpha}\right\} \\
& \leq \sum_{\substack{N\left(\mathfrak{p}^{k}\right) \leq x \\
k \geq 2}}^{*} N(\mathfrak{p})^{\alpha} \sum_{\mathfrak{p}^{k} \| \mathfrak{a}} 1+\sum_{N(\mathfrak{p}) \leq x^{1 / 2}}^{*} N(\mathfrak{p})^{\alpha} \sum_{\mathfrak{p} \mid \mathfrak{a}, \mathfrak{p} \neq P(\mathfrak{a})} 1 \\
& \leq \sum_{\substack{N\left(\mathfrak{p}^{k}\right) \leq x \\
k \geq 2}}^{*} N(\mathfrak{p})^{\alpha} \sum_{N(\mathfrak{a}) \leq x / N\left(\mathfrak{p}^{k}\right)} 1+\sum_{N(\mathfrak{p}) \leq x^{1 / 2}}^{*} N(\mathfrak{p})^{\alpha} \sum_{N(\mathfrak{a}) \leq x / N(\mathfrak{p})} 1 \\
& =O\left\{x \sum_{\substack{N\left(\mathfrak{p}^{k}\right) \leq x \\
k \geq 2}}^{*} N(\mathfrak{p})^{\alpha-1}\right\}+O\left\{x \sum_{N(\mathfrak{p}) \leq x^{1 / 2}}^{*} N(\mathfrak{p})^{\alpha-1}\right\}=O\left(\frac{x^{1+\alpha / 2}}{\log x}\right),
\end{aligned}
$$

where in the last equality we use (5) and Lemma 2. 
Now we make two steps:

(i) We first sum over all $N(\mathfrak{a}) \leq x$ with $N(P(\mathfrak{a})) \leq x / \log ^{M+1} x, M=$ $[(N+1) / \alpha]$, including those with $P(\mathfrak{a}) Q(\mathfrak{a}) \mid \mathfrak{a}, N(Q(\mathfrak{a}))=N(P(\mathfrak{a}))$. Then

$$
\sum_{N(\mathfrak{a}) \leq x} N(P(\mathfrak{a}))^{\alpha} \leq \sum_{N(\mathfrak{a}) \leq x} \frac{x^{\alpha}}{\log ^{\alpha(M+1)} x}=O\left(\frac{x^{1+\alpha}}{\log ^{N+1} x}\right) .
$$

(ii) Next we sum over all $N(\mathfrak{a}) \leq x$ such that $N(P(\mathfrak{a}))>x / \log ^{M+1} x$. Assume $\mathfrak{a}=\mathfrak{p}_{k} \mathfrak{p}_{k-1}^{\alpha_{k-1}} \ldots \mathfrak{p}_{1}^{\alpha_{1}}, N\left(\mathfrak{p}_{k}\right)>N\left(\mathfrak{p}_{k-1}\right) \geq \ldots \geq N\left(\mathfrak{p}_{1}\right)$. Let $\mathfrak{q}=$ $\mathfrak{p}_{k-1}^{\alpha_{k-1}} \ldots \mathfrak{p}_{1}^{\alpha_{1}}$. Noting that $N\left(\mathfrak{p}_{k-1}\right) \leq N(\mathfrak{q})<N\left(\mathfrak{p}_{k}\right)$, if $x$ is large enough, by Lemmas 2 and 3, one has

$$
\begin{aligned}
& \sum_{N\left(\mathfrak{p}_{k} \mathfrak{q}\right) \leq x} N\left(\mathfrak{p}_{k}\right)^{\alpha}=\left.\sum_{N(\mathfrak{q})<\log ^{M+1}} \sum_{N(\mathfrak{q})<\log ^{M+1} x}\left\{\sum_{n=1}^{N} \frac{(n-1) !}{(1+\alpha)^{n}} \cdot \frac{y^{1+\alpha}}{\log ^{n} y}+O\left(\frac{y^{1+\alpha}}{\log ^{N+1} y}\right)\right\}\right|_{x / \log ^{M+1} x} ^{x / N(\mathfrak{q})} \\
= & \sum_{N(\mathfrak{q})<\log ^{M+1} x} \frac{1}{N(\mathfrak{q})^{1+\alpha}} \sum_{n=1}^{N} \frac{(n-1) !}{(1+\alpha)^{n}} \cdot \frac{x^{1+\alpha}}{\log ^{n} x / N(\mathfrak{q})}+O\left(\frac{x^{1+\alpha}}{\log ^{N+1} x}\right) \\
= & \sum_{N(\mathfrak{q})<\log ^{M+1} x} \frac{1}{N(\mathfrak{q})^{1+\alpha}} \sum_{n=1}^{N}\left(\sum_{v=0}^{n-1} \frac{(1+\alpha)^{v} \log ^{v} N(\mathfrak{q})}{v !}\right) \frac{(n-1) !}{(1+\alpha)^{n}} \cdot \frac{x^{1+\alpha}}{\log ^{n} x} \\
& +O\left(\frac{x^{1+\alpha}(\log \log x)^{N}}{\log ^{N+1} x}\right) \\
= & \sum_{n=1}^{N}\left(\sum_{v=0}^{n-1} \frac{(1+\alpha)^{v}}{v !} \sum_{N(\mathfrak{q})<\log ^{M+1} x} \frac{\log N(\mathfrak{q})}{\left.N(\mathfrak{q})^{1+\alpha}\right)} \frac{(n-1) !}{(1+\alpha)^{n}} \cdot \frac{x^{1+\alpha}}{\log ^{n} x}\right. \\
& +O\left(\frac{x^{1+\alpha}(\log \log x)^{N}}{\log ^{N+1} x}\right) \\
= & \sum_{n=1}^{N} \sum_{v=0}^{n-1} \frac{(-1)^{v}(1+\alpha)^{v}}{v !} \zeta_{K}^{(v)}(1+\alpha) \frac{(n-1) !}{(1+\alpha)^{n}} \cdot \frac{x^{1+\alpha}}{\log ^{n} x} \\
& +O\left(\frac{x^{1+\alpha}(\log \log x)^{N}}{\log ^{N+1} x}\right) .
\end{aligned}
$$

In the last equality we use the formulas (cf. [8], Theorem 141)

$$
\sum_{\mathfrak{a}} \frac{1}{N(\mathfrak{a})^{s}}=\zeta_{K}(s), \quad \operatorname{Re} s>1
$$


and

$$
\sum_{\mathfrak{a}} \frac{\log ^{v} N(\mathfrak{a})}{N(\mathfrak{a})^{s}}=(-1)^{v} \zeta_{K}^{(v)}(s), \quad \operatorname{Re} s>1
$$

Noting that $N$ can be any positive integer, by (4), (6) and (7), we complete the proof of Theorem 3 .

\section{References}

[1] K. Alladi and P. Erdős, On an additive arithmetic function, Pacific J. Math. 71 (1977), 275-294.

[2] T. X. Cai, On the mean value estimate of a certain arithmetic function, Chinese Sci. Bull. 29 (1984), 1481-1484 (in Chinese).

[3] - On an additive function on the Gaussian integers, Adv. in Math. (Beijing) 20 (2) (1991), 250

[4] J. M. De Koninck and A. Ivić, Topics in Arithmetical Functions, Notas Mat., Amsterdam, 1980.

[5] —, - The distributions of the average prime divisors of an integer, Arch. Math. (Basel) 43 (1984), 37-43.

[6] P. Erdős and A. Ivić, Estimates for sums involving the largest prime factor of an integer and certain related additive functions, Studia Sci. Math. Hungar. 15 (1980), $183-199$.

[7] E. Landau, Handbuch der Lehre von der Verteilung der Primzahlen, Leipzig, 1909.

[8] - Einführung in die elementare und analytische Theorie der algebraischen Zahlen und der Ideale, Leipzig, 1927.

[9] A. Mercier, Comportement asymptotique de $\sum_{p \leq x} p^{a}\{x / p\}^{k}$, Canad. Math. Bull. 30 (1987), 309-317.

[10] P. Zarzycki, On a certain additive function on the Gaussian integers, Acta Arith. 52 (1989), 75-90.

DEPARTMENT OF MATHEMATICS

HANGZHOU UNIVERSITY

HANGZHOU, P.R. CHINA 